\title{
10001 Microcatheter Re-shaping Using Technical Fusion Image in Coil Embolization: A Technical Note
}

Akihiro Hirayama, Kittipong Srivatanakul, Hideaki Shigematsu, Kazuma Yokota, Takatoshi Sorimachi, and Mitsunori Matsumae

Objective: We report the utility of microcatheter reshaping by referring to fusion images with 3D-DSA and microcatheter 3D images made using non-subtraction and non-contrast (non-SC) rotational images.

Case Presentations: Case 1: The patient was a 74-year-old man who had an internal carotid-anterior choroidal artery bifurcation aneurysm with a tortuous proximal parent artery. The initial attempt to introduce the microcatheter into the aneurysm was unsuccessful. During this unsuccessful microcatheter introduction, we created fusion images with 3D-DSA and microcatheter 3D images by acquiring positional information of the microcatheter using the non-SC method. By reshaping the microcatheter with reference to the fusion images, the direction of the distal end of the microcatheter was reshaped to be in accordance with the long axis of the aneurysm, a shape more suitable for coiling. Case 2: The patient was a 47-year-old man who had an anterior communicating (A-com) artery aneurysm with two daughter sacs. We successfully placed two microcatheters in the direction of each sac to make more stable framing by referring to 3D fusion images after the first microcatheter was positioned. In both cases, microcatheter reshaping was necessary because of the vessel and aneurysm anatomy. We have used this technique successfully in 15 patients, for both ruptured and unruptured aneurysms. The average number of microcatheter reshaping was 1.3 times.

Conclusion: This method provides effective microcatheter reshaping for coil embolization of aneurysms, particularly those with differences between the axis of the parent artery and the vertical axis of aneurysm, or with a tortuous proximal artery.

Keywords > microcatheter shaping, coil embolization, fusion image

\section{Introduction}

Microcatheter shaping is one of the most important factors for achieving safe and effective coiling. In particular, proper positioning of the microcatheter is sometimes difficult in cases of sidewall-type aneurysm such as paraclinoid aneurysms, and in those with differences between the axis of the parent artery and the vertical axis of the aneurysm. In addition, anterior communicating (A-com) artery and/or distally located aneurysm with a tortuous proximal parent

Department of Neurosurgery, Tokai University School of Medicine, Isehara, Kanagawa, Japan

Received: November 1, 2020; Accepted: January 28, 2021 Corresponding author: Akihiro Hirayama. Department of Neurosurgery, Tokai University School of Medicine, 143, Shimokasuya, Isehara, Kanagawa 259-1193, Japan

Email: a-hira@is.icc.u-tokai.ac.jp

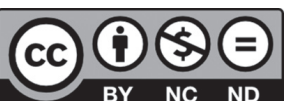

This work is licensed under a Creative Commons Attribution-NonCommercial-

NoDerivatives International License.

(C)2021 The Japanese Society for Neuroendovascular Therapy artery complicates microcatheter placement, and the final alignment and position of the microcatheter tip are often inadequate to achieve sufficient coiling due to the inconsistency of the long axis of the aneurysm. On the other hand, with marked recent progress in image processing, fusion images have been applied in endovascular therapy. ${ }^{1)}$ Fusion images with 3D-DSA and microcatheter 3D images can be created by acquiring spatial information of the microcatheter using rotational angiography without contrast after introducing the microcatheter into the aneurysm, that is, using a non-subtracted and non-contrast (non-SC) method.

We report the utility of microcatheter reshaping with reference to fusion images of 3D-DSA and microcatheter 3D images made using a non-SC method.

\section{Case Presentation}

\section{Materials}

This study investigated 15 aneurysms (8 ruptured aneurysms and 7 unruptured aneurysms) among 33 consecutive aneurysms embolized by a single operator (A.H.) from 


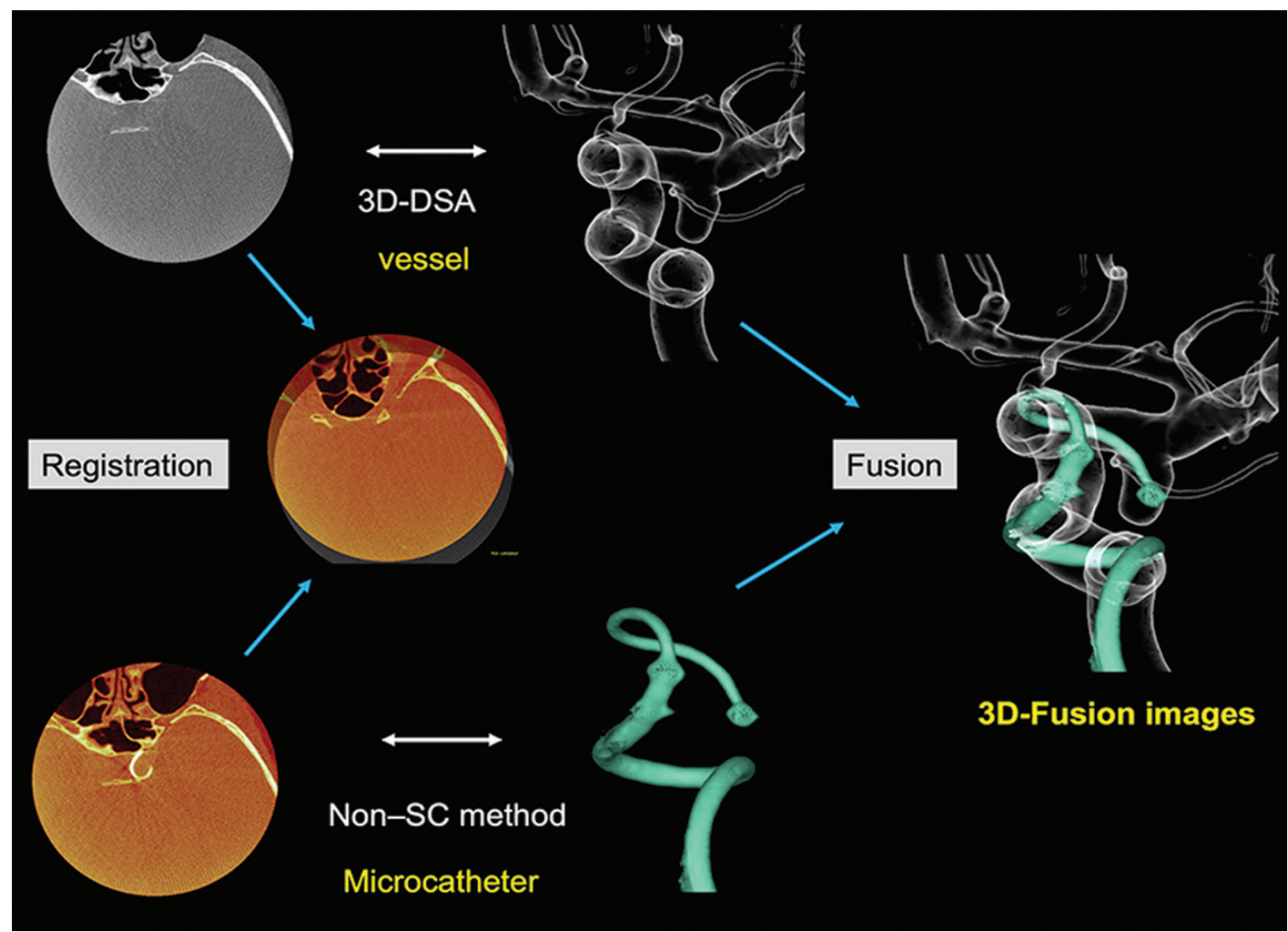

Fig. 1 A workflow image showing the steps for creating images of microcatheter and 3D-DSA. Bone axial source images from initial rotational angiography and intra-procedural bone images acquired by non-SC method are registered on a workstation using spatial registration based on the bone images. Once the registration is completed, images from both volumes can be merged to a single volume. The volume rendering data of the microcatheter are obtained by the non-SC method and can be redone as many times

October 2017 to October 2018 in which the initial microcatheter was not in satisfactory position for coiling.

\section{Workflow for obtaining 3D images}

All patients undergo rotational 3D-DSA to evaluate the morphology of the aneurysm prior to embolization procedure. Reconstruction images are immediately transferred to a workstation (Advantage Workstation 3.1p; GE Healthcare, Waukesha, WI, USA). A microcatheter with manual shaping based on prediction from 3D-DSA or a preshaped microcatheter is introduced into the aneurysm. If the position was not as expected or was unstable, we acquire another rotational image of the microcatheter using a non-SC method. Basically, since the composition of most microcatheters contains some degree of contrast constituent, the microcatheter can be visualized on rotational images without contrast as the operator requires to make the ideal shape. This fusion of the objects from different stages during the procedure allows the operator to understand the path of the microcatheter in the parent artery lumen and the exact position of its tip within the dome of the aneurysm. The time required for creating the fusion images on the workstation is machine- and operator-dependent, in our setting, approximately 1 minute. non-SC: non-subtraction and non-contrast

medium. However, in some types of microcatheter such as the Headway 17 microcatheter (Terumo, Tokyo, Japan), baring low contrast constituent, it is necessary to fill the lumen of the microcatheter with contrast prior to performing a non-SC angiography by injecting manually contrast material to fill the lumen of the microcatheter and stop the irrigation momentarily. Although we believe that this is not a risk for intraoperative rupture, the injection should be done gently and meticulously. These multiplanar reconstruction images of the microcatheter and the initial 3D-DSA are extemporaneously fused on the workstation (Fig. 1). These images are merged using spatial information from both images combined with the semimanual designation of a defined anatomical point such as the petrous and/or sphenoidal bone. The microcatheter 3D images are then fused on 3D-DSA. This allows the 3D fusion image to 

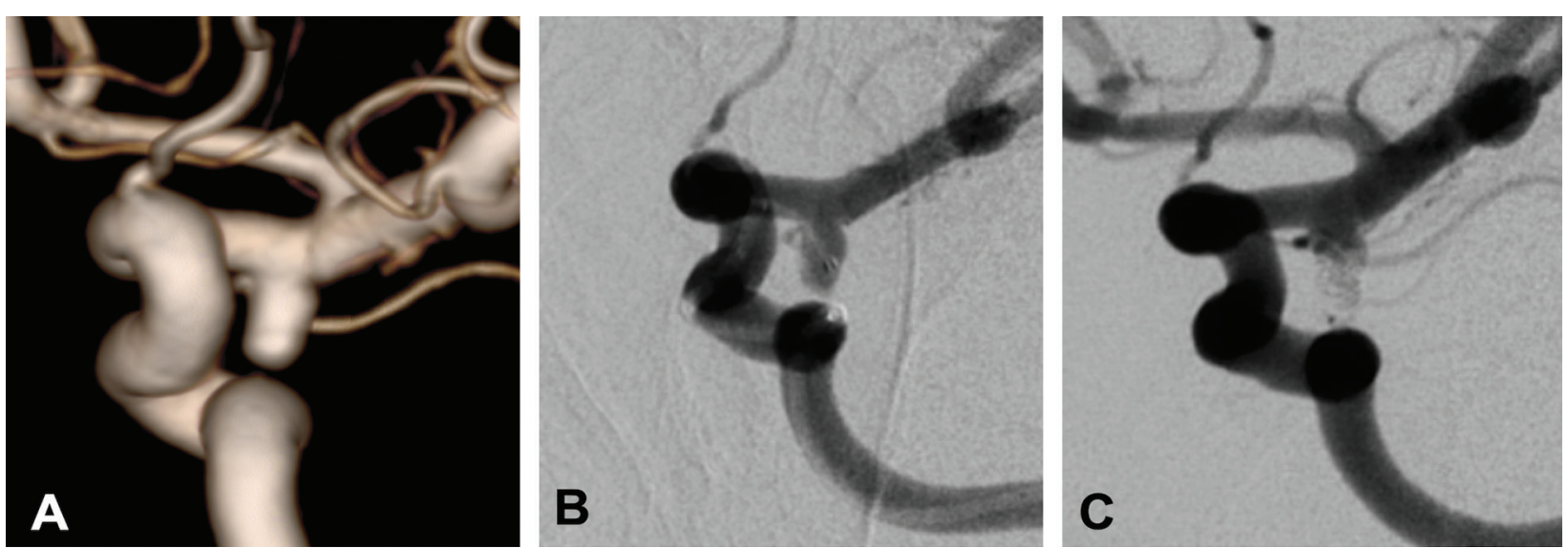

Fig. 2 (A) A preoperative left ICA 3D-DSA image in case 1, demonstrating a tortuous ICA siphon. (B) A left ICA angiogram at working projection, demonstrating a microcatheter with a manual S-shape after the initial introduction into the aneurysm. (C) A postoperative

be observed from arbitrary angles, allowing assessment of where the microcatheter is in contact with the vessel wall and determination of the direction of the microcatheter tip. The microcatheter is then reshaped manually so that the tip would be located appropriately in the aneurysm. After the introduction of the re-shaped microcatheter into the aneurysm, if necessary, we perform another non-SC method to confirm the microcatheter position.

\section{Case 1}

The patient was a 74-year-old man with a history of hypertension. An unruptured saccular aneurysm of the left anterior choroidal artery aneurysm was detected incidentally on MRI. Maximum aneurysm size on MRA was $4.5 \mathrm{~mm}$. On diagnostic angiography, the siphon of the left internal carotid artery (ICA) appeared tortuous (Fig. 2A). The patient was administered aspirin at $100 \mathrm{mg}$ /day for 7 days prior to treatment. Under general anesthesia, heparin was administered in a $3000-\mathrm{U}$ bolus after inserting a $25-\mathrm{cm}$, 6-Fr sheath into the right femoral artery. A 6-Fr guiding catheter was inserted into the right ICA. As an intermediate catheter, a 120-cm, 3.4-/3.2-Fr Tactics (Technocrat, Aichi, Japan) was co-axially placed in C4 of the ICA. A Headway 17 microcatheter, which had been shaped manually according to a $3 \mathrm{D}$ configuration on a surface rendering image of the aneurysm to correspond to both the parent artery configuration and the long axis of the aneurysm, was delivered over a microguidewire into the aneurysm (Fig. 2B). We then acquired fusion images of 3D-DSA and the microcatheter using the non-SC method with the microcatheter filled with contrast medium (Fig. 3A and 3B). After removing the microcatheter, the shape of the tip of the microcatheter was left ICA angiogram at working projection. Coiling of the aneurysm is finished with a slight residual neck and good reservation of the posterior communicating artery. ICA: internal carotid artery

then reshaped with reference to the fusion images and reintroduced to the aneurysm. The new direction of the microcatheter tip was congruent with the long axis of the aneurysm (Fig. 3C and 3D). Coiling of the aneurysm was successfully accomplished using single microcatheter technique with excellent control of the microcatheter (Fig. 2C).

\section{Case 2}

A 47-year-old man developed subarachnoid hemorrhage and presented with sudden-onset disturbance of consciousness. Diagnostic angiography revealed a $6.0-\mathrm{mm}$ A-com aneurysm with two sacs and a wide neck. Under general anesthesia, we performed coiling with the double microcatheter technique. ${ }^{2)}$ An 8-Fr guiding catheter was inserted into the right ICA. As an intermediate catheter, a 118-cm, 6-Fr Cerulean catheter DD6 (Medikit, Tokyo, Japan) was co-axially placed in the petrous portion of the ICA using a 0.035-inch guidewire. An Excelsior SL10 (Stryker, Kalamazoo, MI, USA) $45^{\circ}$ preshaped microcatheter was delivered over a microguidewire to the aneurysm neck (Fig. 4A). We then acquired fusion images by the non-SC method to assess the intra-aneurysmal position and intra-vessel course of the microcatheter (Fig. 4B). For ideal embolization with two microcatheters in this case, the microcatheter tips must be positioned in each sac. With reference to the fusion image of 3D-DSA and the microcatheter, one microcatheter was reshaped at a $90^{\circ}$ angle and inserted into the anterior part of the aneurysm, and another microcatheter with an S-shape was shaped for introduction to the posterior part of the aneurysm (Fig. 4C). Complete coiling was successfully accomplished using a double microcatheter technique with excellent control of 

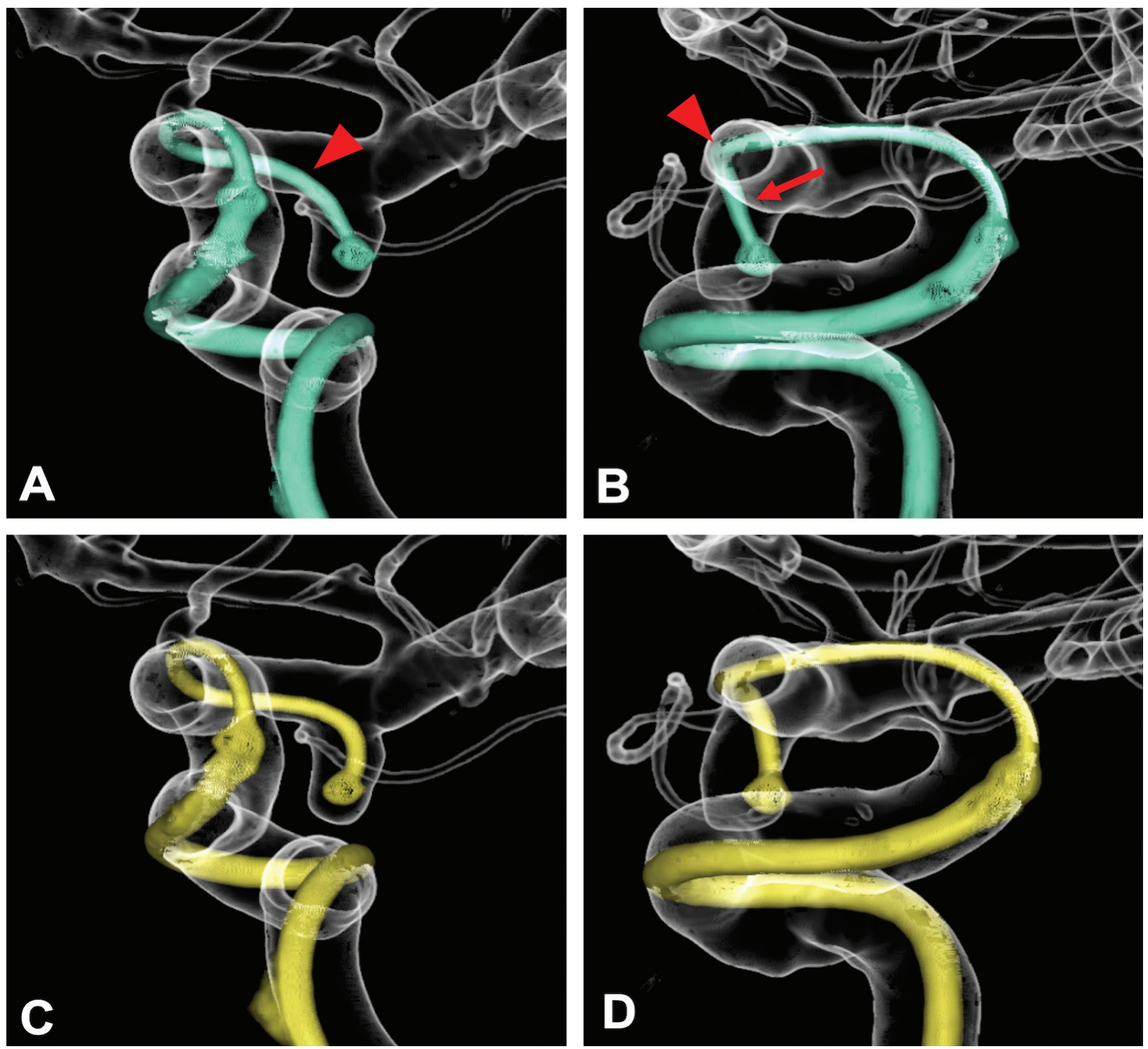

Fig. 3 Fusion images (A: working angle; B: rotation $90^{\circ}$ to the right from the working angle) after the first introduction of the microcatheter demonstrate that the long axis of the aneurysm and the direction of the microcatheter tip are not congruent. The microcatheter is further reshaped by bending the part indicated by the red arrowheads

the microcatheter (Fig. 4D). No procedural complications occurred.

We applied this technique in 15 patients as summarized in Table 1. In 12 of the 15 patients, the microcatheter reached an ideal position with only one reshaping.

\section{Discussion}

In cases of sidewall-type aneurysm or cases with a tortuous proximal parent artery, occasionally the microcatheter cannot be placed in the aneurysm in the way the operator has predicted requiring a complex 3D-shaped microcatheter. In

and adding another curve at the red arrow. Fusion images (C: working angle; $\mathbf{D}$ : rotation $90^{\circ}$ to the right from the working angle) after introduction of the reshaped microcatheter demonstrate good congruence of the long axis of the aneurysm and the direction of the microcatheter tip

recent years, techniques for microcatheter shaping such as referring to a blood vessel model created by $3 \mathrm{D}$ printing before the procedure and re-shaping the microcatheter after placement inside the artery have been reported. ${ }^{3-5)}$ However, a 3D-printed blood vessel model is difficult to prepare in ruptured aneurysm cases and has limited use when readjustment of the shape of the microcatheter is needed. In addition, although microcatheter shaping is reportedly decided in advance according to the location of the aneurysm, ${ }^{6-8)}$ the shape, angle, and length of the microcatheter should be changed according to the individual conditions depending on the direction of the aneurysm axis 

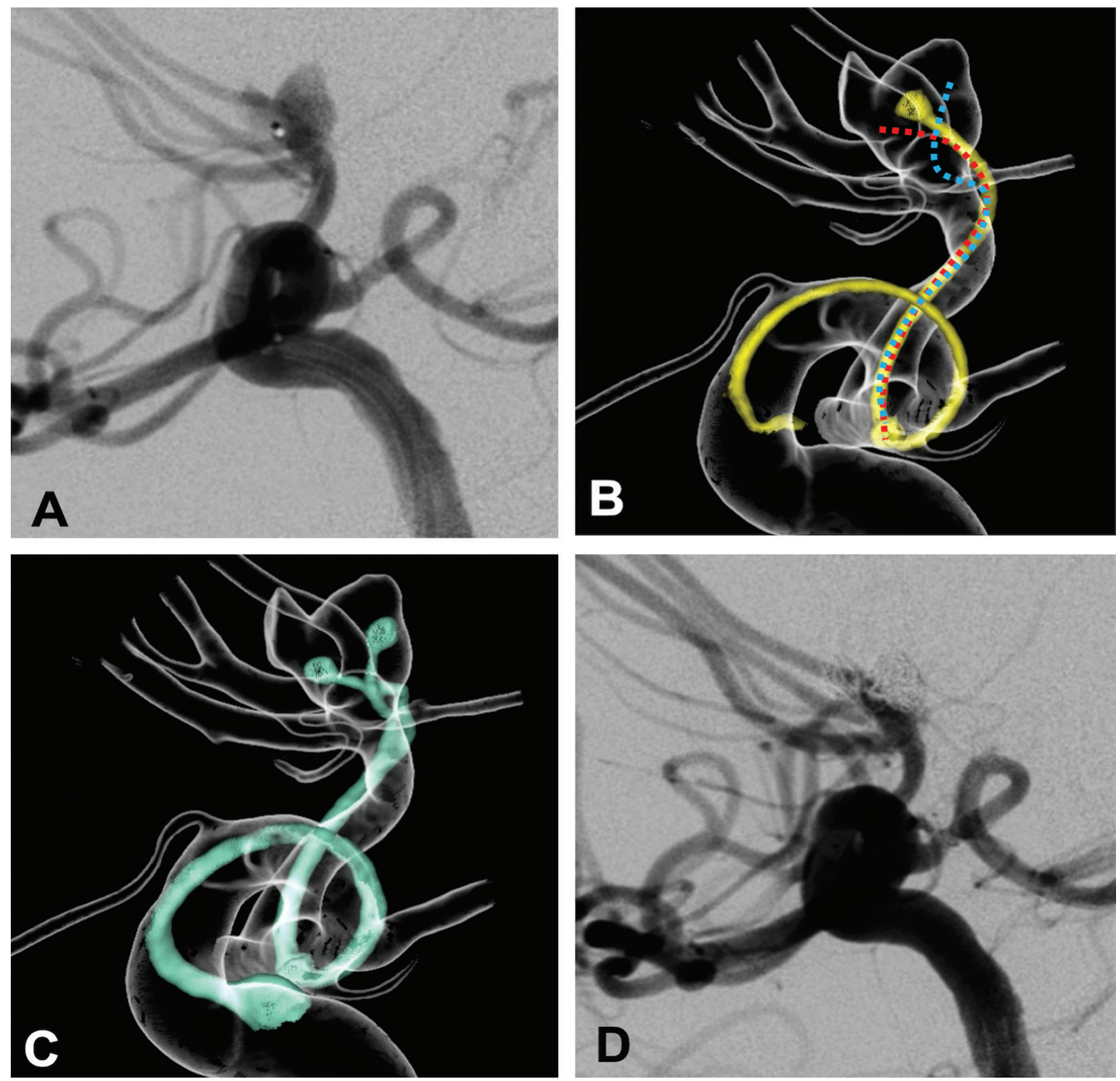

Fig. 4 (A) A right ICA angiogram at working projection after the introduction of the first microcatheter in Case 2. (B) A fusion image after the introduction of the first microcatheter. Red dotted line and blue dotted line demonstrate the ideal microcatheters positions. (C) A fusion image after the introduction of the two microcatheters. The first catheter is then taken out and reshaped according to the information

and the course of the parent artery. Thus, it is necessary to check the microcatheter tip position after first microcatheter introduction and to be able to modify the microcatheter shape if not in the ideal position.

With our method, sites of contact between the microcatheter and blood vessel wall can be observed and the position and orientation of the microcatheter tip in the aneurysm can be examined from every direction on 3D fusion images acquired with low radiation dose and with no contrast medium. This

obtained in (B). A second catheter is shaped accordingly but to point at a direction opposite to the first one. Fusion image after introduction of these two microcatheters demonstrates the position of the microcatheter tip angled appropriately in each aneurysmal sac. (D) A right ICA final angiogram demonstrating complete aneurysmal occlusion. ICA: internal carotid artery

allows better prediction of the ideal shaping of the microcatheter. However, even if the ideal shape of the microcatheter can be predicted from fusion images, problems remain regarding whether this ideal shape can actually be achieved.

In Case 2, showing an A-com aneurysm with a wide neck, the directions of the two microcatheter tips matched the long axes of each aneurysmal sac by reshaping the microcatheters with reference to 3D-fusion images of the position of the initially inserted microcatheter. 
Hirayama $A$, et al.

Table 1 Summary of patient and aneurysm characteristics, treatment method, and outcomes

\begin{tabular}{|c|c|c|c|c|c|c|c|c|}
\hline $\begin{array}{l}\text { Case } \\
\text { No. }\end{array}$ & $\begin{array}{l}\text { Age/ } \\
\text { sex }\end{array}$ & Location & $\begin{array}{l}\text { Ruptured or } \\
\text { unruptured }\end{array}$ & $\begin{array}{l}\text { Aneurysm } \\
\text { size (mm) }\end{array}$ & $\begin{array}{c}\text { Neck } \\
\text { size }(\mathrm{mm})\end{array}$ & $\begin{array}{l}\text { Adjunctive } \\
\text { technique } \\
\text { and DAC }\end{array}$ & $\begin{array}{l}\text { Reshaping } \\
\text { times }\end{array}$ & $\begin{array}{c}\text { Embolization } \\
\text { result }\end{array}$ \\
\hline 1 & $62 / \mathrm{M}$ & ICA-PCoA & Ruptured & $4.3 \times 5.8$ & 2.8 & Single & 1 & $\mathrm{CO}$ \\
\hline 2 & $65 / M$ & ACoA & Unruptured & $6.3 \times 3.3$ & 3.1 & Single/DAC & 2 & $\mathrm{CO}$ \\
\hline 3 & $58 / \mathrm{M}$ & $\mathrm{ACoA}$ & Ruptured & $7.6 \times 5.4$ & 5.1 & Double & 1 & $\mathrm{CO}$ \\
\hline 4 & $72 / \mathrm{F}$ & $\mathrm{ACoA}$ & Ruptured & $3.9 \times 2.6$ & 2.2 & Single & 1 & $\mathrm{CO}$ \\
\hline 5 & $75 / F$ & Paraclinoid & Unruptured & $3.8 \times 4.8$ & 3.5 & Double/balloon & 1 & $\mathrm{RN}$ \\
\hline 6 & $51 / F$ & $\mathrm{ACoA}$ & Ruptured & $7.5 \times 4.4$ & 3.2 & Double & 1 & $\mathrm{CO}$ \\
\hline 7 & $63 / F$ & $\mathrm{ACoA}$ & Ruptured & $7.2 \times 4.1$ & 4.3 & Double & 2 & $\mathrm{RN}$ \\
\hline 8 & $69 / F$ & Paraclinoid & Unruptured & $4.4 \times 5.4$ & 2.9 & Single & 1 & $\mathrm{CO}$ \\
\hline 10 & $77 / F$ & $\mathrm{ACoA}$ & Ruptured & $4.0 \times 8.3$ & 5.0 & Double/DAC & 1 & $\mathrm{CO}$ \\
\hline 11 & $74 / \mathrm{M}$ & ICA-AChA & Unruptured & $3.4 \times 4.5$ & 3.1 & Single/DAC & 2 & $\mathrm{RN}$ \\
\hline 12 & $69 / F$ & MCA & Unruptured & $6.4 \times 5.2$ & 4.2 & Single & 1 & $\mathrm{RN}$ \\
\hline 13 & $77 / F$ & Paraclinoid & Ruptured & $2.4 \times 2.3$ & 1.8 & Single & 1 & RA \\
\hline 14 & $77 / F$ & $\mathrm{ACoA}$ & Unruptured & $6.5 \times 3.3$ & 5.2 & Single/DAC & 1 & $\mathrm{CO}$ \\
\hline 15 & $62 / F$ & $\mathrm{~A} 1$ & Ruptured & $2.7 \times 1.3$ & 1.5 & Single/DAC & 1 & $\mathrm{RN}$ \\
\hline
\end{tabular}

A1: first segment of the anterior cerebral artery; AChA: anterior choroidal artery; ACoA: anterior communicating artery; CO: complete occlusion; DAC: distal access catheter; ICA: internal carotid artery; MCA: middle cerebral artery; PCOA: posterior communicating artery; RA: residual aneurysm; RN: residual neck

This method is also useful from the perspective of operator education, allowing storage of the shaped microcatheter on the workstation providing accurate feedback on the incorrect microcatheter shaping after the procedure. Although this non-SC method requires the same scan time as standard rotational imaging, at $5 \mathrm{~s}$, the exposure radiation dose is decreased by up to approximately two-thirds compared with average biplane 2D-DSA runs, because objects with high contrast such as microcatheters and bone can be acquired with a low radiation dose settings.

Moreover, depending on the material of microcatheter, the microcatheter may need to be filled with contrast medium during imaging. In our series, the SL10 microcatheter, which contains radiopaque material, was imaged with the non-SC method as it, but the non raiopaque Headway microcatheter was imaged after filling up the lumen with contrast. In addition, in case of an aneurysm located in the distal vessel with severely tortuous proximal parent artery, slight misregistration of the 3D-DSA and microcatheter in fusion images is most likely to occur because the blood vessel is stretched by insertion of the microcatheter. However, this slight misregistration of the tip of the microcatheter did not interfere in determining the direction of the microcatheter. On the other hand, with aneurysms located near the skull base such as paraclinoid aneurysms, the bone at the skull base do not interfere with the visualization of the microcatheter as the path of the catheter is inside the lumen of the artery. And in addition, there is less misregistration because the vessel is not displaced due to the fixation to the skull base.

In this study, we reshaped microcatheters to adapt to 15 aneurysms using this method. We consider that the average number of reshapes was relatively low, at 1.3 times. The importance of being able to have a good microcatheter position by proper shaping at the first attempt cannot be overstated. However, if the ideal microcatheter position cannot be obtained initially, fusion images from the non-SC method help in understanding the relationship of the path of the microcatheter to the parent artery. This results in the reduction of the number of microcatheter shaping contributing to less-invasive coil embolization.

\section{Conclusion}

Microcatheter reshaping using fusion images created by a non-SC method yields good positioning and stability of the 
microcatheter for coil embolization of sidewall aneurysms or aneurysms with a tortuous proximal artery.

\section{Disclosure Statement}

All authors have no conflicts of interest to disclose regarding this paper.

\section{References}

1) Suzuki H, Maki H, Taki W: Evaluation of cerebral arteriovenous malformations using image fusion combining three-dimensional digital subtraction angiography with magnetic resonance imaging. Turk Neurosurg 2012; 22: 341-345.

2) Baxter BW, Rosso D, Lownie SP: Double microcatheter technique for detachable coil treatment of large, widenecked intracranial aneurysms. AJNR Am J Neuroradiol 1998; 19: 1176-1178.

3) Ishibashi T, Takao H, Suzuki T, et al: Tailor-made shaping of microcatheters using three-dimensional printed vessel models for endovascular coil embolization. Comput Biol Med 2016; 77: 59-63.

4) Namba K, Higaki A, Kaneko N, et al: Microcatheter shaping for intracranial aneurysm coiling using the 3-dimensional printing rapid prototyping technology: preliminary result in the first 10 consecutive cases. World Neurosurg 2015; 84: $178-186$.

5) Ohshima $T$, Imai $T$, Goto $S$, et al: A novel technique of microcatheter shaping with cerebral aneurysmal coil embolization: in vivo printing method. JNET J Neuroendvasc Ther 2016; 11: 48-52.

6) Kwon BJ, Im SH, Park JC, et al: Shaping and navigating methods of microcatheters for endovascular treatment of paraclinoid aneurysms. Neurosurgery 2010; 67: 34-40; discussion 40.

7) Xiaochuan H, Xiaoyun S, Youxiang L, et al: Endovascular treatment of unruptured A1 segment aneurysms. Interv Neuroradiol 2013; 19: 186-194.

8) Jia ZY, Song YS, Sheen JJ, et al: Loop microcatheter technique for coil embolization of paraclinoid aneurysms. Acta Neurochir (Wien) 2018; 160: 1755-1760. 